\title{
Another Feather in the Cap: Launch of International Edition and Activation of Online Manuscript Management System
}

W hen we assumed office in January 2008, we started with a dream. The dream was to achieve newer heights for our beloved journal, and to take it to the top; at least in India and South East Asia region. We are inching closer to this vision by delivering the "best" of art and science suited to a scientific publication. Abrief outline of what we have achieved in last two and half years follows:

As promised at the outset(1), we have kept our commitment of timely decision on manuscripts, making life easier for the authors, added international reviewers to our database, and tried our level best to be transparent (and approachable) in the peer review process. We have moved to research based editorials; and publishing at least one state of the art review article/perspective every month. Besides this, there is more material now for practitioners to help in their day to day working that include guidelines/ recommendations/consensus statements, focus, evidence based medicine etc. Another first for Indian Pediatrics is the compendium of all the practice oriented articles in a book form, released recently. The Journal received its first Impact Factor in mid 2008, which was 0.756 . Within a year, it increased by almost $30 \%$ to 0.956 . By the time, you are reading this piece; the 2010 list of impact factor would have also been released. We are now ranked fourth amongst all the biomedical journals in India. Our website attracts 6-8 lakh hits per month and is rated as number 1 in its category. For the young faculty, Indian Pediatrics has started a unique venture in the form of workshops on 'Art and Science of Paper Writing' to inculcate the culture of publishing in upcoming researchers and teachers. For the authors, we have started the facility of e-publication ahead of print (and getting it indexed with PubMed) so that they do not have to wait for long to see their work in print.
All this could not have been possible without the love, affection and support of our authors, reviewers, more than 18000 subscribers of print version, and lakhs of people in more than 50 countries who view our electronic version to access its contents. We are proud to say that we have achieved all this maintaining the editorial independence and not letting the personal, financial, or other relationships affect our decisions.

And now, we proudly announce the launch of the International edition of Indian Pediatrics (for overseas distribution). The contents will essentially remain the same (sans advertisements); however, the cover will be different. Simultaneously, we are delighted to announce that on 19th May 2010, the online manuscript submission, tracking and peer review system Editorial Manager has been successfully implemented for the journal and is now open for new submissions at https:// www.editorialmanager.com/inpe/. The system has been configured and tailored according to the needs of 'Indian Pediatrics'. The channel is now open for users after extensive in-house testing and training at the journal office. From 1st July, 2010, we have made it compulsory for authors to submit all their manuscripts through this system only. The peer review and decision process would also be managed through this system.

The International Edition and Editorial Manager are the result of a unique collaboration with Springer, a global scientific, technical and medical publisher. Springer will now host Indian Pediatrics on its website and will have the exclusive rights to distribute the International edition. However, unlike its contemporaries, the journal will continue to host its contents on its website and the open access status of the journal will be maintained for authors and 
readers who access the journal through www.indianpediatrics.net, which will remain fully functional as before with the added link for online submission. Indian Pediatrics can also be accessed through springer webpage by clicking on to www.springer.com/medicine/pediatrics/journal/ 13312

\section{Features of Online Manuscript Management SYSTEM}

Though Indian Pediatrics is receiving and processing the manuscripts online for many years, the online manuscript management system (MMS) aims to make your work as author and reviewer easier. The system enables faster review times and transparency of workflow, such as the ability to keep track of the manuscript. Authors need to do a one-time registration in the system by clicking on the 'Register' button from the menu at the above website and enter the requested information. Upon successful registration, authors are sent an automatic e-mail with instructions to verify the registration. Authors can start submitting the manuscripts from this point onwards. They can also respond to reviewers' comments and revise the manuscript using the same system. The biggest advantage, however, is to track the progress of all manuscripts submitted to the journal at the click of the mouse. The system is userfriendly and follows a step-by-step process for manuscript submission, revision and tracking, with instructions prominently displayed at each step. There is an easy process of retrieving the password from the system if one forgets the same.

For reviewers, the system offers the option of agreeing or not agreeing to review a manuscript, after seeing the abstract on their mail. All existing reviewers of the journal are already registered with the system. Once they agree to review, the reviewers can access the manuscript and submit comments using the same system. The system links the references in the manuscript to the PubMed and allows reviewers to check their authenticity and style. The reviewer also gets to know of the final decision on the manuscript. Reviewers can submit the manuscripts to the journal as authors using the same username and password.

For editors, a variety of features are available which help in more organized and faster workflow. The system enables faster review times and transparency, such as the ability to keep track of individual editor's and reviewer's performance. The facility for automatic reminders and organization of manuscripts according to their current status, minimizes the paperwork and need to send individual mails to authors and reviewers.

\section{Challenges Ahead}

Technology comes with its own caveats. With the start of the MMS, the journal expects increase in number of submissions from different parts of the world. With increase in submissions, the rejection rate is likely to further increase because of the limited number of pages available for publication per month. The authors need to be careful in submitting the manuscripts taking care to correctly enter the necessary details. They need to ensure that the details that are entered in the system while submitting the manuscript are same as those on the title page of the manuscript. Authors need to refer to the 'Instructions for Authors', which have been updated at the journal website, for details and information on how to prepare the manuscript to meet the journal's requirements. Minimum errors in the submission process are likely to expedite the review and publication process. We hope that this new MMS will make your work as author and reviewer easier.

The story does not end here. Picture abhi baki hai mere dost..... it would not be inappropriate here to quote Robert Frost to express our sentiments "the woods are lovely, dark and deep; but I have promises to keep, and miles to go before I sleep... and miles to go before I sleep." There are many more horizons waiting to be conquered. Jai Hind, Jai IAP.

\section{Piyush Gupta, Editor-in-Chief; and Dheeraj Shah, Associate Editor; Indian Pediatrics, New Delhi, India. editorinchief.indianpediatr@gmail.com}

Funding: None.

Competing interests: None stated.

\section{REFERENCE}

1. Gupta P. Indian Pediatrics: looking ahead. Indian Pediatr 2008; 45: 9-10. 